\title{
On the influence of lipid-induced optical anisotropy for the bioimaging of exo -or endocytosis with interference microscopic imaging
}

\author{
Dylan Marques ${ }^{1,2}$, Adelaide Miranda $\mathrm{PhD}^{2}$, Ana G. Silva Prof ${ }^{1}$, Peter \\ R. T. Munro $\mathrm{PhD}^{3}$, and Pieter A. A. De Beule PhD, \\ pieter.de-beule@inl.int ${ }^{2}$ \\ ${ }^{1}$ Departamento de Física, Faculdade de Ciências e Tecnologia, \\ Universidade Nova de Lisboa, Campus de Caparica, Caparica, \\ P-2829-516, Portugal \\ ${ }^{2}$ Applied Nano-Optics Group, Department of Life Sciences, \\ International Iberian Nanotechnology Laboratory, Braga 4715-330, \\ Portugal \\ ${ }^{3}$ Department of Medical Physics and Biomedical Engineering, \\ University College London, Malet Place, Gower Street, London, \\ WC1E 6BT, United Kingdom
}

April 13, 2018

\begin{abstract}
Some implementations of interference microscopy imaging use digital holographic measurements of complex scattered fields to reconstruct three-dimensional refractive index maps of weakly scattering, semi-transparent objects, frequently encountered in biological investigations. Reconstruction occurs through application of the object scattering potential which assumes an isotropic refractive index throughout the object. Here, we demonstrate that this assumption can in some circumstances be invalid for biological imaging due to the presence of lipid-induced optical anisotropy. We show that the nanoscale organization of lipids in the observation of cellular endocytosis with polarized light induces a significant change in far field scattering. We obtain this result by presenting a general solution to Maxwell's equations describing light scattering of core-shell particles near an isotropic substrate covered with an anisotropic thin film. This solution is based on an extension of the Bobbert-Vlieger solution for particle scattering near a substrate delivering an exact solution to the scattering problem in the near field as well as far-field. By applying this solution to study light scattering by a lipid vesicle near a lipid bilayer, whereby
\end{abstract}


the lipids are represented through a biaxial optical model, we conclude through ellipsometry concepts that effective amounts of lipid-induced optical anisotropy significantly alter far field optical scattering in respect to an equivalent optical model that neglects the presence of optical anisotropy.

The taking in of matter by a living cell by invagination of its membrane to form a vacuole, i.e. endocytosis, and the process of vesicles budding off membranes for the transport of membrane bound secretory vesicles to the extracellular matrix, i.e. exocytosis, exemplify fundamental processes in biology. In-vivo detection of both exo- and endocytosis represents a formidable challenge to image capture technology due to the speed of the event, the size of the lipid vesicles and optical contrast available. Polarization resolved Total Internal Reflectance Fluorescence Microscopy (TIRFM) has been proven a powerful technique for the dynamic ([?]) observation of exocytosis. In particular, TIRFM has proven capable of characterizing localized topological changes of the plasma membrane upon exocytosis. Within a different context ([?]) illustrated how combined fluorescence confocal microscopy and video Differential Interference Contrast (DIC) microscopy data can jointly contribute to the study of dynamic membrane remodelling processes during nerve growth.

The image formation process of biological cells in widefield white light optical microscopy can be expected to be influenced by structured optical anisotropy caused by lipid membranes ([?]). An effective relation between the amount of lipid membrane optical anisotropy and optical microscopy images remains elusive and phase sensitive microscopy techniques such as e.g. interference microscopy imaging approaches can be expected to depend on lipid optical anisotropy, although it is not entirely clear how. Such Label-free imaging techniques have an advantage over fluorescence microscopy since they do not require the introduction of highly stable fluorescent molecules capable of reporting vesicle tracking. Furthermore, the scattering crosssections encountered when performing label free imaging are typically several orders of magnitude higher than that of fluorescence microscopy. DIC is a widely used interference microscopy technique that makes use of orthogonal orientations of linearly polarized light to measure the interference of light originating from sample locations separated by a well-defined shear distance. It allows one to derive a linear phase map of the sample ([?]). However, providing a meaningful biophysical understanding of the contrast origin in such a phase map remains challenging ([?]). In order to fully predict the DIC microscopic image formation process, and thus link it to the state of the imaged object, it is necessary to investigate how light is scattered by optically anisotropic structures.

Here, we present the formal solution, satisfying Maxwell's equations, for light scattered by core-shell particle with an optically biaxial shell above an isotropic substrate covered with an anisotropic thin film. We apply this 
solution to the case of light scattering by a liposome above a lipid bilayer, embedded in an aqueous environment, taking into account the lipid induced optical anisotropy. Furthermore, we study how light scattering by such samples alters due to the presence of optical anisotropy, with the help of ellipsometric concepts. Our numerical simulations detail how far-field light scattering during cellular exo- and endocytosis is perturbed by the presence of lipid-induced optical anisotropy.

\section{Light scattering of a liposome suspended above a lipid membrane}

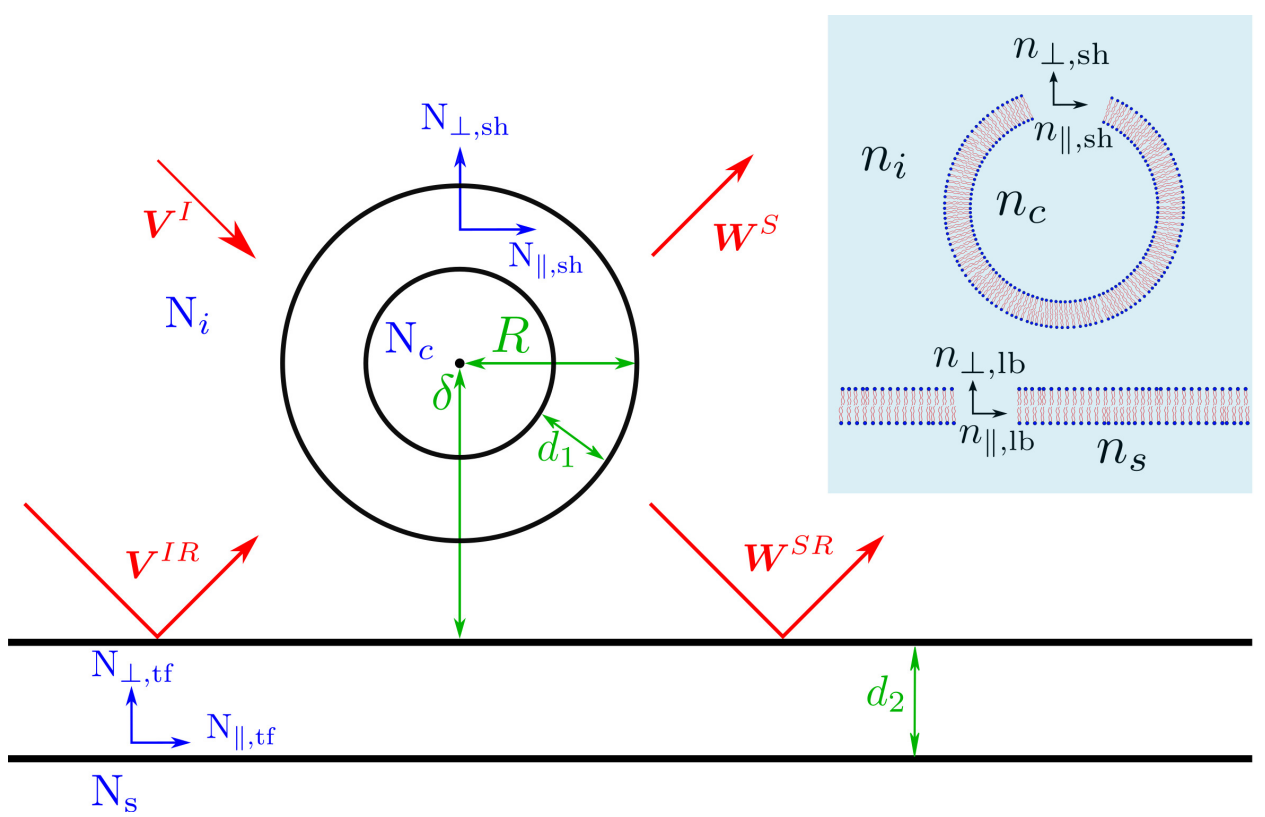

Figure 1: Light scattering of a core-shell particle with an optically biaxial shell near a substrate covered with an optically biaxial thin film. The electromagnetic waves represented by spherical Debye potentials are represented in red; geometrical dimensions are indicated in green and RI regions indicated in blue. The top right insert represent an equivalent model for a lipid vesicle above a lipid bilayer.

Figure 1 depicts the general system to which we present an analytical solution: a core-shell particle consisting of a solid isotropic core characterized by the complex optical constant $\mathrm{N}_{c}$ and biaxial shell represented by complex optical constants $\mathrm{N}_{\perp \text {,sh }}$ and $\mathrm{N}_{\|, \mathrm{sh}}$, suspended in an isotropic medium $\left(\mathrm{N}_{i}\right)$ near a substrate $\left(\mathrm{N}_{s}\right)$ covered by a biaxial thin film $\left(\mathrm{N}_{\perp, t \mathrm{tf}}\right.$ and $\left.\mathrm{N}_{\|, \mathrm{tf}}\right)$. Further parameters considered in our model system are overall particle radius $R$, particle to thin film distance $\delta$, shell thickness $d_{1}$ and thin film thickness $d_{2}$. 
The solution satisfying Maxwell's equation for light scattered by a sphere near a substrate was presented by Bobbert and Vlieger in 1986 ([?]) and subsequently evaluated experimentally through ellipsometry by Bobbert et al. ([?]) Their method relies on the formal solution represented by

$$
\mathbf{W}^{\mathbf{S}}=(1-\mathbf{B} \cdot \mathbf{A})^{-1} \cdot \mathbf{B} \cdot\left(\mathbf{V}^{\mathbf{I}}+\mathbf{V}^{\mathbf{I R}}\right),
$$

whereby the incident and reflected waves are represented by spherical Debye potentials $\mathbf{V}^{\mathbf{I}}, \mathbf{V}^{\mathbf{I R}}, \mathbf{W}^{\mathbf{S}}$, and $\mathbf{W}^{\mathbf{S R}}$ as indicated in figure 1. Matrices $\mathbf{A}$ and $\mathbf{B}$ characterize reflection of spherical waves by the substrate and light scattering by the particle respectively. This solution was originally applied to solid spheres on an isotropic substrate. Using the T-matrix approach, this has been extended to axisymmetric and even arbitrary shape particles $([?, ?])$. Also, non-homogeneous particles with isotropic and biaxial coatings $([?, ?])$ have been considered. It should be noted that a simplified approximate solution has been presented by Videen ([?, ?]), also applied in reference ([?]), assuming that the scattered field reflecting off the surface and interacting with the particle is incident upon the surface at near-normal incidence. However, great care should be exercised by application of this approximation, especially for metallic particles, as pointed out by ([?])

In order to establish the solution for the scattered light depicted in figure 1 we extend the solution presented in reference ([?]) by modifying the Fresnel reflection coefficients contained within matrix $\mathbf{A}$ with the Fresnel reflection coefficient for a substrate covered with a biaxial anisotropic thin film ([?]). Here, we evaluate the solution for the total light reflected from a surface density of identical core-shell particles as detailed by Bobbert et al. ([?]). The numerical simulations reported below have been obtained by extending the implementation of the Bobbert-Vlieger theory found in the SCATMECH library developed by Thomas A. Germer ([?]).

A suitable optical model for cellular exo- or endocytosis representing a liposome above a lipid bilayer in an aqueous environment is represented in the top right of figure 1 . The main difference with the general model is that the attenuation coefficient for all media, i.e. lipids and aqueous medium, is considered zero: the complex valued optical constant $\mathrm{N}$ therefore reduces to the real valued Refractive Index (RI) n. It should be noted that in this investigation the RI are considered non-dispersive. For the biaxial model an average $\mathrm{RI}$ is defined according to

$$
N_{a v g}^{2}=\frac{2 N_{\|}^{2}}{3}+\frac{N_{\perp}^{2}}{3}
$$

Unless otherwise stated, all default parameters as illustrated in 1 for the modelling of sparsely seeded core-shell particles on a surface according to reference ([?]) are listed in table 1 . 
Table 1: Default simulation parameters.

\begin{tabular}{l|c||l|c} 
Model parameter & Value & Model Parameter & Value \\
\hline \hline$\theta$ & $70^{\circ}$ & $\lambda$ & $0.488 \mu \mathrm{m}$ \\
\hline $\mathcal{S}$ & $1 \mu \mathrm{m}^{-2}$ & $n_{i}=n_{s}=n_{c}$ & 1.333 \\
\hline$n_{\|, \mathrm{sh}}=n_{\|, \mathrm{lb}}$ & 1.450 & $n_{\perp, \mathrm{sh}}=n_{\perp, \mathrm{lb}}$ & 1.460 \\
\hline$n_{\text {avg,sh }}=n_{\text {avg,lb }}$ & 1.453 & $d_{1,2}$ & $5 \mathrm{~nm}$ \\
\hline \hline
\end{tabular}

In this work, we use ellipsometry concepts to link observable properties of scattered light with the microscopic optical properties of the sample. Ellipsometry typically determines the amplitude ratio of light reflected off a sample polarized parallel and perpendicular to the plane of incidence. This ratio is typically defined as

$$
\frac{r_{p}}{r_{s}}=\tan \Psi(\lambda, \theta) e^{-i \Delta(\lambda, \theta)},
$$

and is determined entirely by ellipsometry angles $\Psi$ and $\Delta$ respectively

In this study, we are interested in the difference in scattering between an optical model based on an isotropic sample and an optical model taking into account the lipid induced optical anisotropy. Hence, we define ellipsometry angles $\delta \Delta=\Delta-\Delta_{\text {avg }}$ and $\delta \Psi=\Psi-\Psi_{\text {avg }}$ as the differences of ellipsometry angles for the anisotropic and isotropic (avg) optical models, respectively. In the isotropic (avg) case the ordinarily anisotropic refractive indices are all replaced by their corresponding average refractive index defined through equation 2. Furthermore, we define the ellipsometry angle differences $D_{\Psi}$ and $D_{\Delta}$ for Angle Of Incidence (AOI) resolved ellipsometry measurements at incident angles $\theta_{1}, \theta_{1}+\delta \theta, \ldots, \theta_{1}+n \delta \theta$.

$$
\begin{aligned}
& D_{\Psi}=\sum_{i=1}^{n} \frac{\left|\delta \Psi\left(\theta_{i}\right)\right|}{n} \\
& D_{\Delta}=\sum_{i=1}^{n} \frac{\left|\delta \Delta\left(\theta_{i}\right)\right|}{n} \\
& d=\frac{1.22 \lambda}{\mathrm{NA}_{\mathrm{obj}}+\mathrm{NA}_{\mathrm{cond}}}
\end{aligned}
$$

\section{Results}

Figure 2 depicts modelling results $D_{\Psi}$ and $D_{\Delta}$ in absence of the liposome, i.e. based on the Fresnel reflection coefficients for an isotropic substrate covered with a biaxial thin film only. We can distinguish three distinct regions according to the impact of lipid film optical anisotropy being strong, weak 
and negligible, respectively: 1) $\left.n_{i} \sim n_{s} \sim n_{\text {avg }, l b}, 2\right) n_{i} \sim n_{s} \nsim n_{\text {avg,lb}}$ and 3) $n_{i} \nsim n_{s}$. We observe a maximum for $D_{\Psi}$ and $D_{\Delta}$ when $n_{i}=n_{s}=n_{a v g, l b}$. The AOI resolved spectroscopic ellipsometry curves for $\Psi$ and $\Delta$ underlying three example points in the above mentioned regions are shown in figure 2 (bottom left) and (bottom right) respectively. Given that aqueous media have a RI of $\sim 1.33$, we can conclude that the small optical anisotropy of a lipid bilayer has a relatively significant impact on the far-field scattering polarization properties, in comparison to an isotropic model equivalent. If the incoming media is aqueous $\left(n_{i}=1.33\right)$ while the substrate medium is glass-like $\left(n_{s}=1.45\right)$, one can deduce from the colour scale of the top row of figure 2 that the impact of lipid optical anisotropy on the ellipsometry $\Delta$ angle will be substantially stronger in respect to changes in ellipsometry $\Psi$ angle. This observation can be considered useful for observation of cellular lipid membranes near a glass substrate. It should, however, be noted that the overall reflected intensity drops to zero in zone one where $n_{i} \sim n_{s} \sim$ $n_{\text {avg }, l b}$ since this represents a homogeneous sample, so while the effect under investigation becomes larger it becomes increasingly challenging to observe experimentally.

Ellipsometry modelling of liposomes with radii $R$ of $50 \mathrm{~nm}, 150 \mathrm{~nm}$ and $250 \mathrm{~nm}$ above a lipid bilayer are illustrated in figure 3 for a wavelength (top) and AOI (bottom) resolved ellipsometry respectively. For spectroscopic ellipsometry the model predicts a $\sim 2.5^{\circ} \Psi$ shift upon consideration of the lipid bilayer optical anisotropy for light scattering at an AOI $70^{\circ}$, largely independent of the liposome diameter. On the contrary, optical anisotropy has a progressively stronger impact on $\Delta(\lambda)$ for larger liposome diameters. From the AOI resolved ellipsometry $\Psi$ angle, we note that the influence of lipidinduced optical anisotropy becomes more pronounced for increasing AOI with a $\sim 1^{\circ}$ discrepancy between anisotropic and correspondingly isotropic averaged model at an AOI of $30^{\circ}$ as compared to $\sim 3^{\circ}$ at AOI $80^{\circ}$. Several obstacles might prevent the experimental observation of the theoretically predicted curves reported by figure 3 , including sample polydispersity, vesicle aggregation, Poisson statistics of the particle density, capacity to obtain well defined isotropic and anisotropic shells around a perfectly homogeneous core and other sample homogeneities. However, figure 3 does indicate a significant capacity of a small 0.01 shell anisotropy modifying the phase of a transmitted wave which is critical for the observation with interference microscopic imaging.

Finally, we show in figure 4 the evolution of ellipsometry angles $\Psi$ and $\Delta$ for liposomes with $100 \mathrm{~nm}$ radius for a change in liposome to lipid bilayer distance $\delta-R$, which express the change in scattering expected to occur over the course of an exo- or endocytosis process. In accordance with Figure 3, lipid-induced optical anisotropy induces a $\sim 2^{\circ}$ shift in $\Psi$. Interestingly, the ellipsometry angle $\Delta$ exhibits a sinusoidal-like behaviour with a period of $\sim 700 \mathrm{~nm}$ and an amplitude of $\sim 1.5^{\circ}$. Inclusion of the lipid optical 

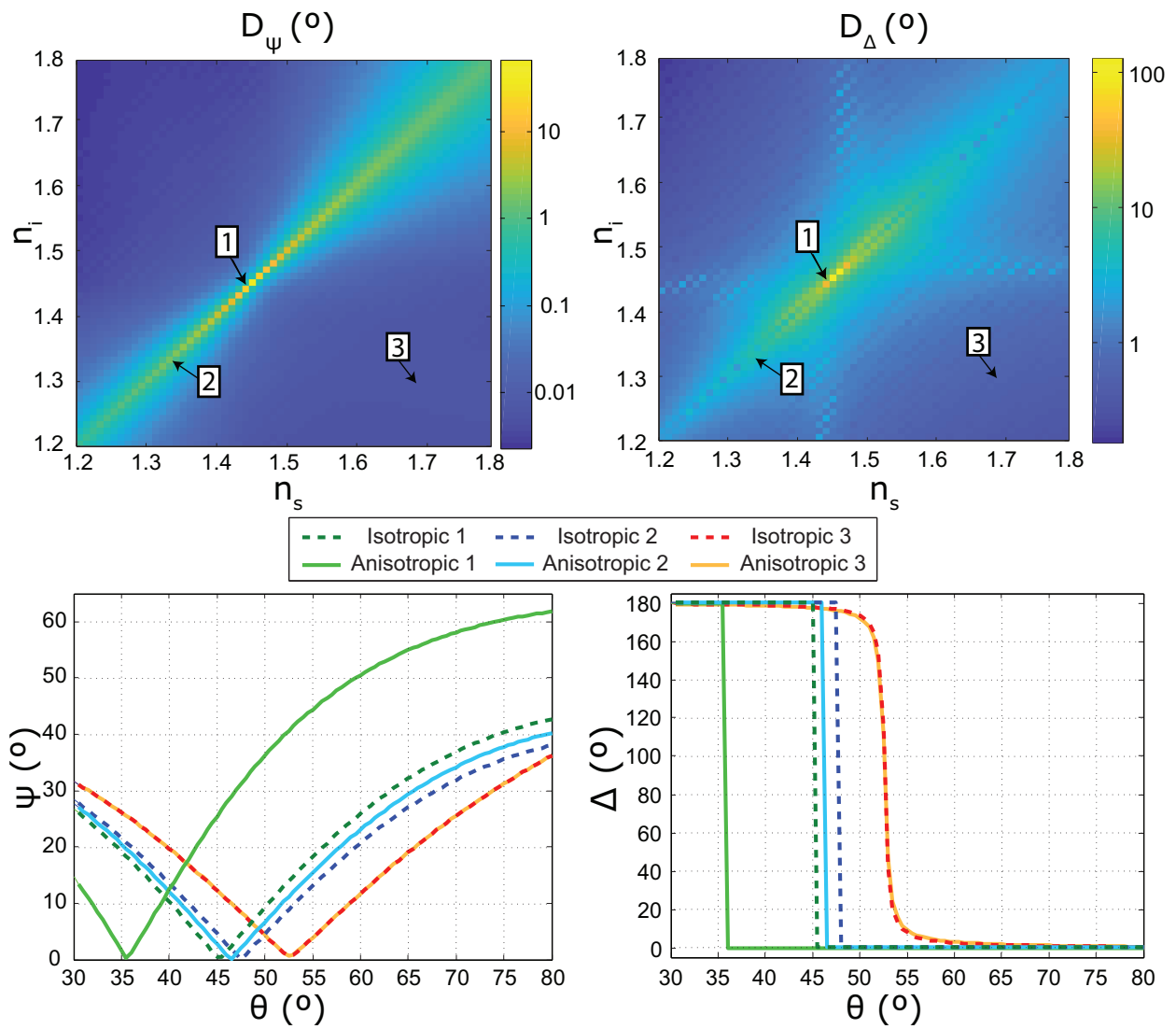

Figure 2: Optical modelling without presence of the lipid sphere: $D_{\Psi}$ (top left) and $D_{\Delta}$ (top right) for AOI resolved ellipsometry from $30^{\circ}$ to $80^{\circ}$ for different values of the incident and substrate refractive indices respectively; AOI resolved $\Psi$ (bottom left) and $\Delta$ (bottom right) profiles for anisotropic lipid thin film and the isotropic counterpart based on the average RI.

anisotropy reduces this amplitude to $\sim 1^{\circ}$ while maintaining the sinusoidal period. Similarly to the discussion following figure 2, figure 4 suggests that optical anisotropy plays a role in the observation of cellular exo- or endocytosis with interference microscopic imaging.

\section{Conclusion}

We have demonstrated that small values, i.e. 0.01, of lipid-induced optical anisotropy alter the far-field optical scattering properties for a liposome above a lipid bilayer with respect to a corresponding all isotropic optical model based on the corresponding average RI. We achieved this through an extension to the Bobbert-Vlieger solution of particle scattering near a substrate. This work effectively demonstrates that lipid optical anisotropy 

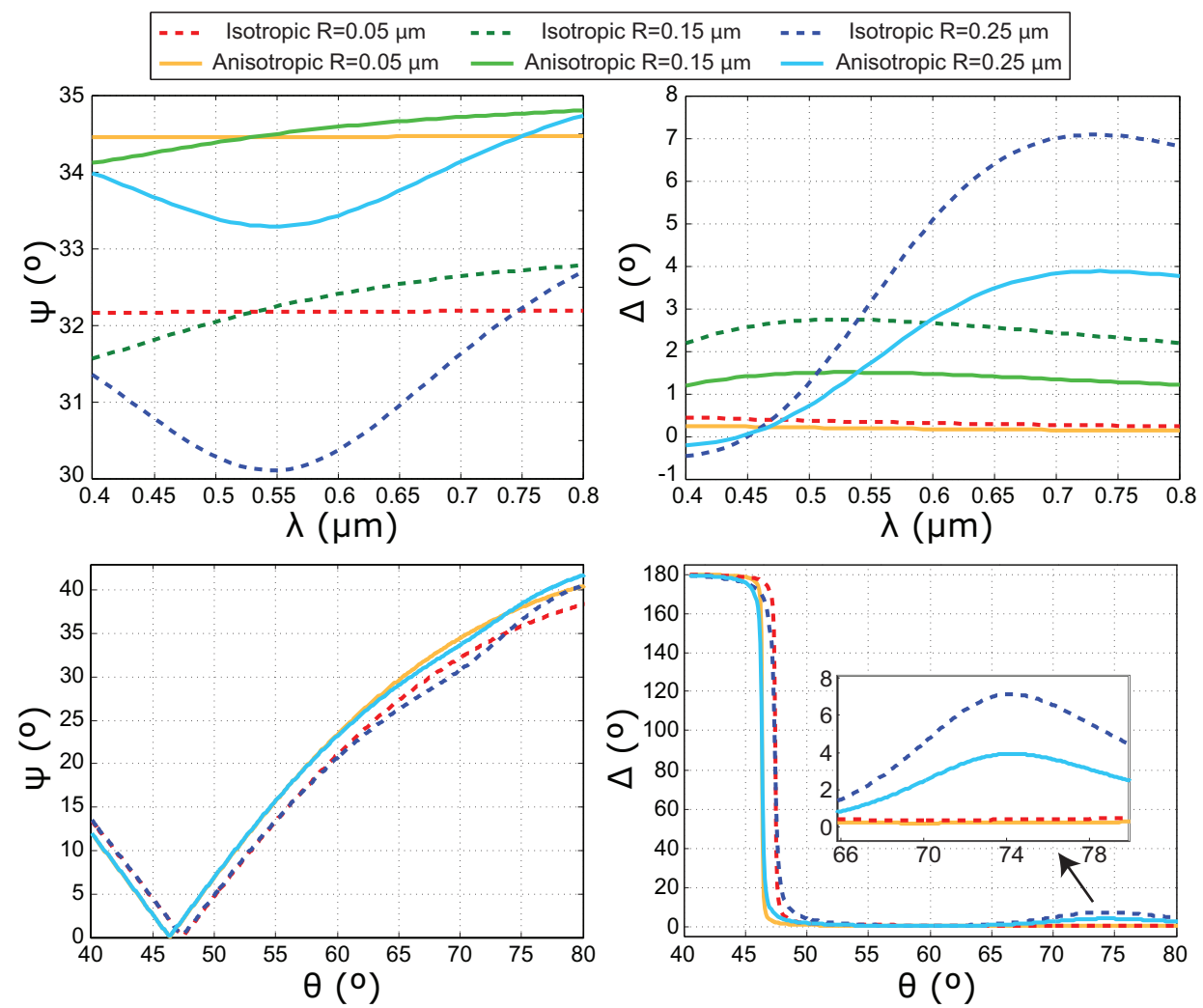

Figure 3: Ellipsometry angles $\Psi$ (left) and $\Delta$ (right) for wavelength (top) and AOI (bottom) resolved ellipsometry of liposome with radii of $50 \mathrm{~nm}$, $150 \mathrm{~nm}$ and $250 \mathrm{~nm}$ above a lipid bilayer $(\delta=R)$. Results for $R=0.15 \mu \mathrm{m}$ have been omitted from the bottom graphs due to limited curve separation.

can be expected to play an important role in the microscopic observation of cellular exo- or endocytosis by phase sensitive interference microscopy imaging including DIC microscopy, more recently introduced approaches relying on digital holography referred to as Quantitative Phase Imaging (QPI) ([?, ?]) and tomographic phase microscopy ([?]). For the latter, we believe that one should be cautious with the interpretation of refractive index maps obtained using prevailing reconstruction techniques which are based upon the concept of isotropic scattering potential introduced by ([?]):

$$
\chi(x, y, z)=\beta_{0}^{2}\left[n^{2}(x, y, z)-n_{m}^{2}\right],
$$

where $\beta_{0}^{2}=2 \pi / \lambda_{0}$ represents the propagation constant in free space, $n(x, y, z)$ the isotropic refractive index (RI) distribution of the object and $n_{m}$ the refractive index of the surrounding medium. This formulation is derived by assuming an isotropic sample RI distribution and so the impact of an 


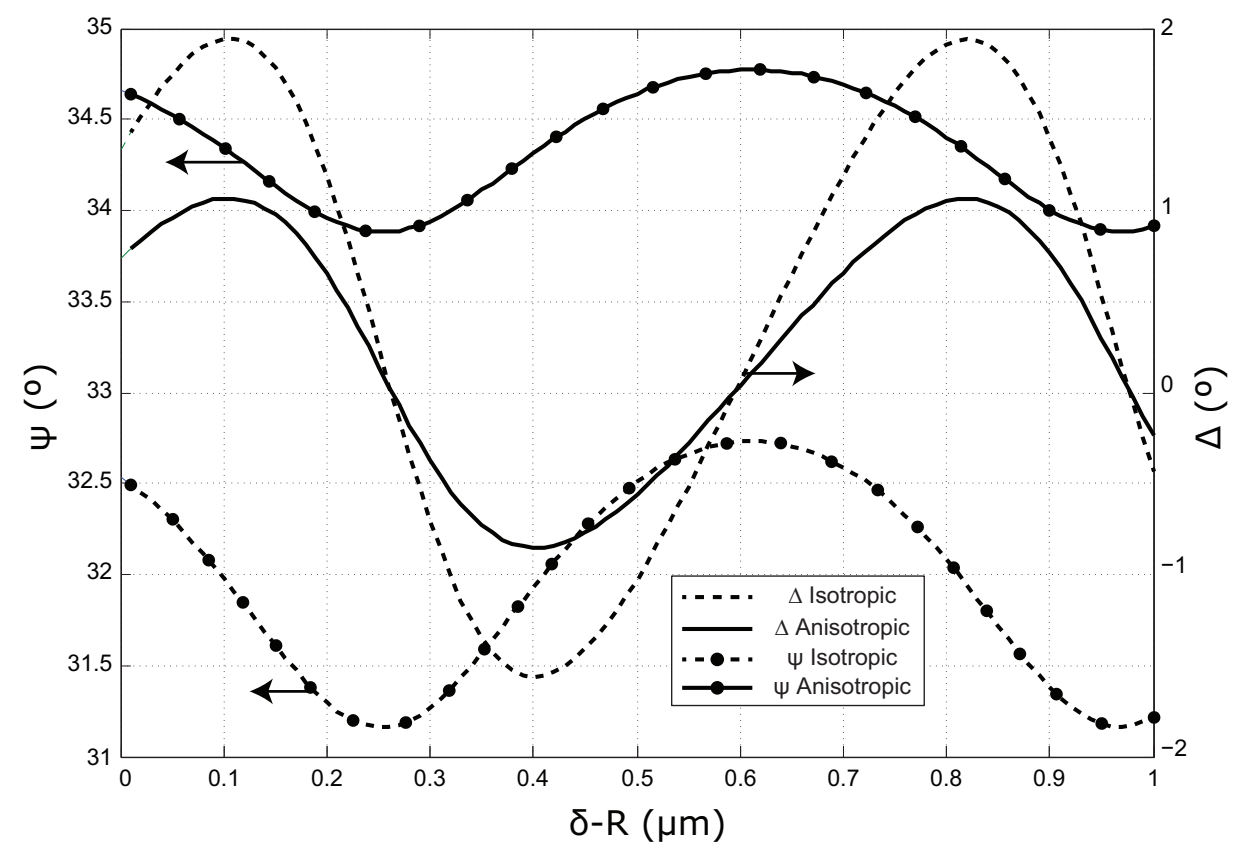

Figure 4: Ellipsometry angles $\Psi$ and $\Delta$ of a liposome above a lipid bilayer for varying distance between the liposome and lipid bilayer $\delta-R$.

anisotropic sample RI is currently not understood. Additionally, we would like to point out that, provided lipid-induced optical anisotropy is known to be temperature dependant as reported for example by ([?]), our work concomitantly therefore predicts that image formation of cellular exo- or endocytosis can be temperature-dependant.

Experimental validation of the light scattering modelling results presented above in an ellipsometry configuration are challenging due to the small liposome radius, limited reflectivity and state-of-the-art achievable signal-to-noise ratio for ellipsometry measurements at the solid-liquid interface ([?]). Hence, we propose to perform experimental validation of the impact of lipid-induced optical anisotropy on interference microscopy in a DIC imaging set-up. This, however, requires integration of the scattering modelling presented here with a DIC image formation model compatible with high Numerical Aperture (NA) image formation by taking into account the full vectorial nature of the electromagnetic field near imaging focus ([?]). Experimental validation will furthermore require careful construction of stable nanoparticle-lipid constructs, representing the focus of ongoing work. 


\section{acknowledgements}

We would like to thank Dr. Thomas Germer for advice on using the SCATMECH polarization modelling library.

\section{funding}

Norte2020, Norte's Regional Operational Programme 2014-2020, Grant Number: NORTE-01-0145-FEDER-000019

\section{to whom correspondance should be addressed}

Pieter A. A. De Beule PhD, pieter.de-beule@inl.int, Applied Nano-Optics Group, Department of Life Sciences, International Iberian Nanotechnology Laboratory, Braga, 4715-330, Portugal 